\title{
Ultrastructural view of a promising anti TNF- $\alpha$ agent on hepatic ischaemia reperfusion injury
}

\author{
Kilicoglu B ${ }^{1}$, Kilicoglu SS $\mathrm{S}^{2}$, Tuncal S ${ }^{1}$, Onalan $\mathrm{AK}^{1}$, Celepli $\mathrm{S}^{1}$, Tasova V ${ }^{1}$, Namuslu $\mathrm{M}^{3}$, Erdemli E
}

General Surgery Department of Ankara Training and Research Hospital, Ankara. kilicoglubulent@yahoo.com

\begin{abstract}
BACKGROUND: Ischemia reperfusion causes injury to the liver cells during transplantation, trauma and emergency surgery. We investigated whether the anti TNF- $\alpha$ agent, etanercept, can reduce injury in an animal model of ischemia reperfusion owing to the fact that TNF-a plays a critical role in the process of inflammation. MATERIALS AND METHODS: Thirty rats were divided into three groups: sham (Group 1), control (Group 2), etanercept $(5 \mathrm{mg} / \mathrm{kg}$ ) treatment (Group 3). Ischemia-reperfusion model was carried out by clamping the hepatic pedicle for $45 \mathrm{~min}$ and then reperfusing the liver for $60 \mathrm{~min}$. Etanercept $(5 \mathrm{mg} / \mathrm{kg})$ was injected intraperitoneally $5 \mathrm{~min}$ prior to reperfusion. At the end of the procedures, blood and liver tissue samples were obtained for biochemical and histopathological assessment.

RESULTS: Control and treatment groups showed significant differences in hepatic function tests, plasma and tissue oxidative stress parameters. Samples in the control group histopathologically showed morphologic abnormalities specific to ischemia reperfusion. Histomorphologic findings in the treatment groups showed similar features as the sham group.

CONCLUSIONS: Our evidence suggests that TNF- $\alpha$ plays a key role in liver ischemia reperfusion injury and etanercept may provide a novel therapeutic approach for patients undergoing liver surgical procedure (Tab. 3, Fig. 4, Ref. 22). Text in PDF www.elis.sk. KEY WORDS: liver, ischemia-reperfusion, liver injury, anti TNF- $\alpha$.
\end{abstract}

\section{Introduction}

Depending on the severity of ischemia reperfusion (I/R), liver injury remains an unresolved problem in liver surgery, transplantation and hemorrhagic shock which leads to acute liver failure and remote organ damage. Over the past decade, there is unfortunately still no guarantee for adequate liver function despite medical advances. As the knowledge of the pathogenesis of the hepatic $I / R$ has increased, treatments directed at spesific components of the immune system have been developed.

The pathophysiology of the hepatic IR includes a number of mechanisms that contribute to various degrees in the overall injury. These mechanisms involve alterations in energy metabolism, microcirculatory disturbances, neutrophil accumulation, formation of proinflammatory mediators, such as endothelin and tumor necrosis factor-alpha (TNF- $\alpha$ ), and reactive oxygen species generation. Although substantial progress has been made in elucidating

${ }^{1}$ General Surgery Department of Ankara Training and Research Hospital, Ankara, ${ }^{2}$ Histology-Embriology Department of Ufuk University Faculty of Medicine, Ankara, ${ }^{3}$ Biochemistry Department of Turgut Özal University Faculty of Medicine, Ankara, and ${ }^{4}$ Histology-Embriology Department of Ankara University Faculty of Medicine, Ankara

Address for correspondence: B. Kilicoglu, MD, General Surgery Department of Ankara Training and Research Hospital, Ulucanlar Altindağ, Ankara, Turkey.

Phone: +903125953448 the mechanisms of IR injury, inflammatory response is clearly recognized as the key mechanism of injury ${ }^{[1]}$. The release of inflammatory mediators with the activation of Kupffer cells is the consequence of ischemia and reperfusion mediated hepatic injury. Stimulation of primed Kupffer cells by complement factors causes continuous activation of these macrophages $(2,3)$.

Primary cytokines, such as TNF- $\alpha$ and IL-1, are generated by Kupffer cells during reperfusion. These cytokines recruit and activate $\mathrm{CD} 4^{+} \mathrm{T}-$-lymphocytes in the liver during the early reperfusion period. $\mathrm{CD} 4^{+} \mathrm{T}$-lymphocytes can produce mediators, such as TNF- $\beta$, IFN- $\gamma$, and granulocyte colony stimulating factor, which amplify Kupffer cell activation and promote neutrophil recruitment into the liver. In addition, TNF- $\alpha$ and IL- 1 are potent inducers of hepatic chemokine synthesis, and under certain circumstances TNF- $\alpha$ can directly trigger apoptotic cell death. Because of the central role of TNF- $\alpha$ in promoting the inflammatory response at initial levels, suppressing the formation of TNF- $\alpha$ or neutralizing it with antibodies proved to be highly effective in attenuating reperrfusion injury (1).

Tumour necrosis factor (TNF) is a cytokine that elicits a large number of biological effects. TNF actions are initiated by binding to two distinct TNF receptors TNFR1 and TNFR2, with TNFR1 mediating the majority of the TNF effects (4). TNFR1 is expressed constitutively on the surface of all cell types as a trimer of $55 \mathrm{kDa}$ subunits, and TNFR2 is expressed in activated immune cells as a trimer of $75 \mathrm{kDa}$ subunits. The binding of TNF to its receptors 


\section{1-607}

leads to the activation of several second messengers like protein kinase A, protein kinase $\mathrm{C}$ and mitogen-activated protein (MAP) kinases ERK1 and ERK2. As these protein kinases have been implicated also in the mechanism of signal transduction by many other extracellular stimuli, it seems unlikely that the activation of these is sufficient to account for the distinctive pattern of activities that are induced by TNF $(5,6)$. Among the proinflammatory cytokines, TNF- $\alpha$ is a potent cytokine that exerts pleiotropic inflammatory and immunological functions by triggering synthesis of downstream targets such as interleukin-6 (5).

Because of the critical role of TNF- $\alpha$ in inflammation cascade, anti TNF- $\alpha$ has been identified as a key therapeutic agent for treating inflammatory diseases such as rheumatoid arthritis, ankylosing spondylitis, psoriasis, and inflammatory bowel disease. Multiple TNF- $\alpha$ agents have been approved by the U.S. Food and Drug administration (FDA) to manage and treat several immunological conditions and are prescribed by specialty clinics such as rheumatology, dermatology, and gastroenterology, with an increasing frequency and for longer duration of use (7).

Etanercept is one of the antiTNF- $\alpha$ agents approved by FDA. It is a dimeric fusion protein consisting of two ligand-binding domains of the soluble human TNF receptor (sTNFR2) linked to the FC fragment of human immunoglobulin G1 (IgG1). It binds to TNF- $\alpha$ and renders it biologically unavailable and thus ineffective (8).

Recent progress in the understanding of inflammation that drives hepatic injury in IR has directed our attention to proinflammatory cytokines secreted by macrophages as targets for therapy, including TNF- $\alpha$. The aim of this article is to investigate the anti TNF- $\alpha$ agent, etanercept in IR model of liver and to comment on its potential role histopathologically with ultrastructural imaging.

\section{Materials and methods}

\section{Animals}

Thirty Wistar-Albino female rats, weighing $250 \pm 30 \mathrm{~g}$ obtained from the Bioscience unit of our Institution were housed in standard conditions and had free access to commercial chow and water. All procedures described here had prior approval from local animal ethics committee.

\section{Study groups and surgical procedures}

Rats were randomly divided into three groups each including 10 animals: SHAM group, control group (ischemia-reperfusion) and treatment groups (etanercept group). Animals were anesthetized by intramuscular injection of $80 \mathrm{mg} / \mathrm{kg}$ ketamine hydrochloride (Ketalar ${ }^{\circledR}$; Parke- Davis, Istanbul, Turkey) and $20 \mathrm{mg}$ / kg xylasine (Rompun ${ }^{\circledR}$, Bayer, Istanbul, Turkey). After the abdomen was shaved and disinfected, a midline incision was done and rats underwent either sham surgery or ischemia-reperfusion. Ischemia was carried out by clamping for 45 min with a microvascular "bulldog" clamp of hepatic pedicle. After this period of occlusion, the clamps were removed. In a separate set of experiments, liver ischemia and reperfusion injury was induced in anti TNF- $\alpha$ treated with Etanercept ( $5 \mathrm{mg} / \mathrm{kg}$ i.p.) $5 \mathrm{~min}$ prior to reperfusion.
Etanercept was given by i.p. injection after reconstitution with 1 $\mathrm{mL}$ sterile bacteriostatic water for injection. The various groups of rats were killed at $60 \mathrm{~min}$ for histological examination of the liver and for biochemical studies, as described below.

\section{Biochemical analyses}

Plasma alanine aminotransferase (ALT), aspartate aminotransferase (AST) and amylase levels were measured for evaluating the liver functions by using Olympus Au 640 autoanalyzer. To assess oxidative injury, malondialdehyde (MDA) levels, glutathione peroxidase (GSH-Px) enzyme activities were determined in the blood samples and malondialdehyde (MDA) levels and glutathione peroxidase (GSH-Px) enzyme activities were determined in the pancreas samples.

\section{Evaluation of oxidative stress}

After sacrification of the animals, liver samples were removed and kept on an ice bath until homogenization. The sample of liver was first washed with distilled water, the tissues were homogenized in (20\% w/v, approximately $1 \mathrm{~g}$ in $5 \mathrm{ml}$ for each) physiological saline, then they were centrifuged 4000xg for $15 \mathrm{~min}$ and upper clear supernatants were used in the assays. All procedures were performed at $+4{ }^{\circ} \mathrm{C}$ throughout the experiments. Protein level of the clear supernatants was studied by Lowry's method (9), and then they were adjusted to equal concentrations before the other analyses. All results were expressed as unit/mg protein for those of pancreas tissues. Malondialdehyde (MDA) level (nmol/mg) and glutathione peroxidase (GSH-Px) enzyme activity ( $\mathrm{mlU} / \mathrm{mg}$ ) were measured in the supernatants.

MDA level was measured by thiobarbituric acid reactive substances method. GSH-Px activity was measured by following changes in NADPH absorbance at $340 \mathrm{~nm}$.

\section{Histological evaluation}

The histopathological analyses were carried out in HistologyEmbryology Department of Ufuk University and Ankara University, Faculty of Medicine.

For light microscope analyses, tissue samples from the liver were obtained from all animals. The liver samples were fixed in $10 \%$ neutral buffered formalin solution for 2 days. Tissues were washed in flowing water and were dehydrated with rising concentrations of ethanol $(50 \%, 75 \%, 96 \%$, and $100 \%)$. After dehydration, specimens were put into xylene to obtain transparency and were then infiltrated with and embedded in paraffin. Embedded tissues were cut into $5 \mu \mathrm{m}$ thick sections by Leica RM 2125 RT. Systematically randomly selected sections were stained with hematoxylin and eosin (H\&E) Periodic Acid-Schiff (PAS). Histopathological examinations were performed and photographed with Olympus DP71.

For transmission electron microscope (TEM) analyses, samples were fixed with phosphate buffered (pH: 7.3) $2.5 \%$ glutaraldehyde and $2 \%$ PFA mixture solution for 2 hours at room temperature. They were washed with phosphate-buffered saline solution 
(PBS) (pH: 7.3) and were fixed with $1 \%$ osmium tetraoxide for 2 hours as secondary fixation. After washing, they were embedded in Araldite 6005 and were cut with Leica EM FCS (Wien- Austria) ultramicrotome. $1 \mu \mathrm{m}$ semi-thin sections were stained by toluidine blue-Azur II to select the region of interest for the following procedures. $60-70 \mathrm{~nm}$ thin sections were stained with uranil acetate and lead citrate. They were examined and photographed using a LEO 906 E TEM (80 kV- Oberkochen- Germany).

\section{Statistical analysis}

Data analysis was performed using SPSS 15.0 package program. Data were presented as mean \pm standard deviation. Differences among the groups were evaluated by One-Way ANOVA or Kruskal Wallis variance analysis, where appropriate. When the p-value from the variance analysis was statistically significant, Mann Whitney U multiple comparison test was used to assess which group differs from the others. Furthermore, Student's t-test variance analysis was used for evaluation of the histopathological results. All results were accepted as statistically significant when $\mathrm{p}<0.05$.

\section{Results}

\section{Biochemical analyses}

The results of liver function tests of the groups are summarized in Table 1. There was a significant difference between the SHAM group and control group according to the levels of AST, ALT $(\mathrm{p}<0.001$ for AST and ALT). There was a significant difference between the control group and treatment group according to the levels of AST, ALT $(p<0.05)$. There was no significant difference between the sham group and treatment groups according to the levels of AST, ALT ( $p=0.96)$.

The plasma levels of MDA and GSH-Px are summarized in Table 2. There was a significant difference between the control

Tab. 1. Liver function tests.

\begin{tabular}{lcc}
\hline Groups & AST $(\mathrm{U} / \mathrm{L})$ & ALT $(\mathrm{U} / \mathrm{L})$ \\
\hline SHAM Group & $101.60 \pm 17.25^{*}$ & $39.60 \pm 3.22^{*}$ \\
Control Group & $367.60 \pm 23.28$ & $151.20 \pm 41.29$ \\
Treatment Group & $128.60 \pm 28.32^{*}$ & $89.40 \pm 33.19^{\#}$ \\
\hline ALT - alanine aminotransferase, AST - aspartate aminotransferase, ${ }^{*} \mathrm{p}<0.001$, \\
${ }^{*} \mathrm{p}<0.05$
\end{tabular}

Tab. 2. Plasma oxidative stress activities of the groups.

\begin{tabular}{lcc}
\hline Groups & MDA (unit/mL) & GSH-Px (unit $/ \mathrm{mL}$ ) \\
\hline SHAM Group & $1.66 \pm 0.25^{*}$ & $362.06 \pm 8.45^{*}$ \\
Control Group & $3.10 \pm 0.97$ & $207.22 \pm 7.89$ \\
Treatment Group & $1.77 \pm 0.54^{*}$ & $377.06 \pm 6.45^{*},{ }^{*}$ \\
\hline MDA - malondialdehyde, GSH-Px - glutathione peroxidase enzyme activity, \\
${ }^{*} \mathrm{p}<0.05,{ }^{*} \mathrm{p}<0.001$
\end{tabular}

Tab. 3. Tissue oxidative stress activities of the groups.

\begin{tabular}{lcc}
\hline Groups & MDA $(\mathrm{nmol} / \mathrm{mg})$ & GSH-Px $(\mathrm{mlU} / \mathrm{mg})$ \\
\hline SHAM Group & $0.34 \pm 0.05$ & $36.42 \pm 12.21$ \\
Control Group & $0.55 \pm 0.11$ & $21.47 \pm 5.88$ \\
Treatment Group & $0.37 \pm 0.07^{\#}$ & $32.44 \pm 8.10^{\#}$ \\
\hline MDA-malondialdehyde, GSH-Px - glutathione peroxidase enzyme activity, ${ }^{\#} \mathrm{p}<0.05$
\end{tabular}

group and other groups in to the MDA and GSH-Px levels $(\mathrm{p}<$ 0.05 ). There was no significant difference between the sham group and treatments groups in the MDA and GSH-Px levels ( $p>0.05)$.

The liver tissue levels of MDA and GSH-Px are summarized in Table 3. There was no statistically significant difference between the SHAM group and control group according to MDA and GSHPx levels $(p>0.05)$. There was statistically significant difference between the control group and treatment group according to MDA and GSH-Px levels ( $\mathrm{p}<0.05$ for MDA and GSH-Px).

\section{Light microscopic evidence}

Group I (control) showed no alteration to the natural structure of liver (Fig. 1 A1, A2). The sinusoids can just be seen as palestained spaces between the plates of liver cells (Figure 1A2). The hepatic acinus is a more physiologically useful model of liver structure and lies between two or more terminal hepatic venules and blood flows from the portal tract through the sinusoids to the venules. The acinus is divided into zones 1,2 , and 3 . The glycogen in hepatocytes was found PAS-positive in the hepatocyte cytoplasm of all zones (Fig. 1 A3).

Group II (ischaemia-reperfusion), showed multiple and extensive areas of portal inflammation with a moderate increase in the level of inflammatory cell infiltration (Fig. 1 B1). The remarkable congestion was extending over a wide area of all sections (Fig. 1 B2). Sinusoidal dilatation was marked. Hepatocytes necrosis was most remarkable in zone 3. The PAS stained sections showed heterogeneous distribution of glycogen in the hepatic acinus. In consequence of the glycolysis there was a significant glycogen depletion which was conspicuously existing in the zone 3 (Fig. 1 B3).

In group III (etanercept treated), the pathological changes indicating ischaemia-reperfusion injury were ameliorated by the etanercept treatment. The marked congestion in the distended sinusoids seen in the ischaemia-reperfusion group was negligible and the glycogen dissociation was homogenous (Fig. 1 C1, C2, C3).

\section{Electron microscopic evidence}

Group I represented the hepatocytes reflecting their extraordinary range of biosynthetic and degradative activities, with the hepatocyte cytoplasm crowded with organels like rough endoplasmic reticulum, smooth endoplasmic reticulum, lysosomes and rich of mitochondria. Glycogen rosettes were present in variable numbers depending on nutritional status. The plasma membranes of adjacent hepatocytes especially close to canalicules were tightly bound and the intercellular space width was usual. Hepatocytes were exposed to the sinusoids lined by a discontinuous layer of sinusoid lining cells. Erythrocytes could be seen within the sinusoids. The extent of space of Disse between the sinusoid lining cells and the hepatocyte surface was of regular size (Fig. 2).

In Group II, cytoplasmic appearance of whole of the hepatocytes composing the liver tissue was dense like compressed with the organelles. The parenchyma was edematous and filled with necrotic cells. We investigated the corruption of the hepatocytes plasma membrane undergoing necrosis (Fig. 3A). The swelling of mitochondria with the fuzzy cristae was marked in the cytoplasm of hepatocytes. The glycogen droplets in the hepatocytes 

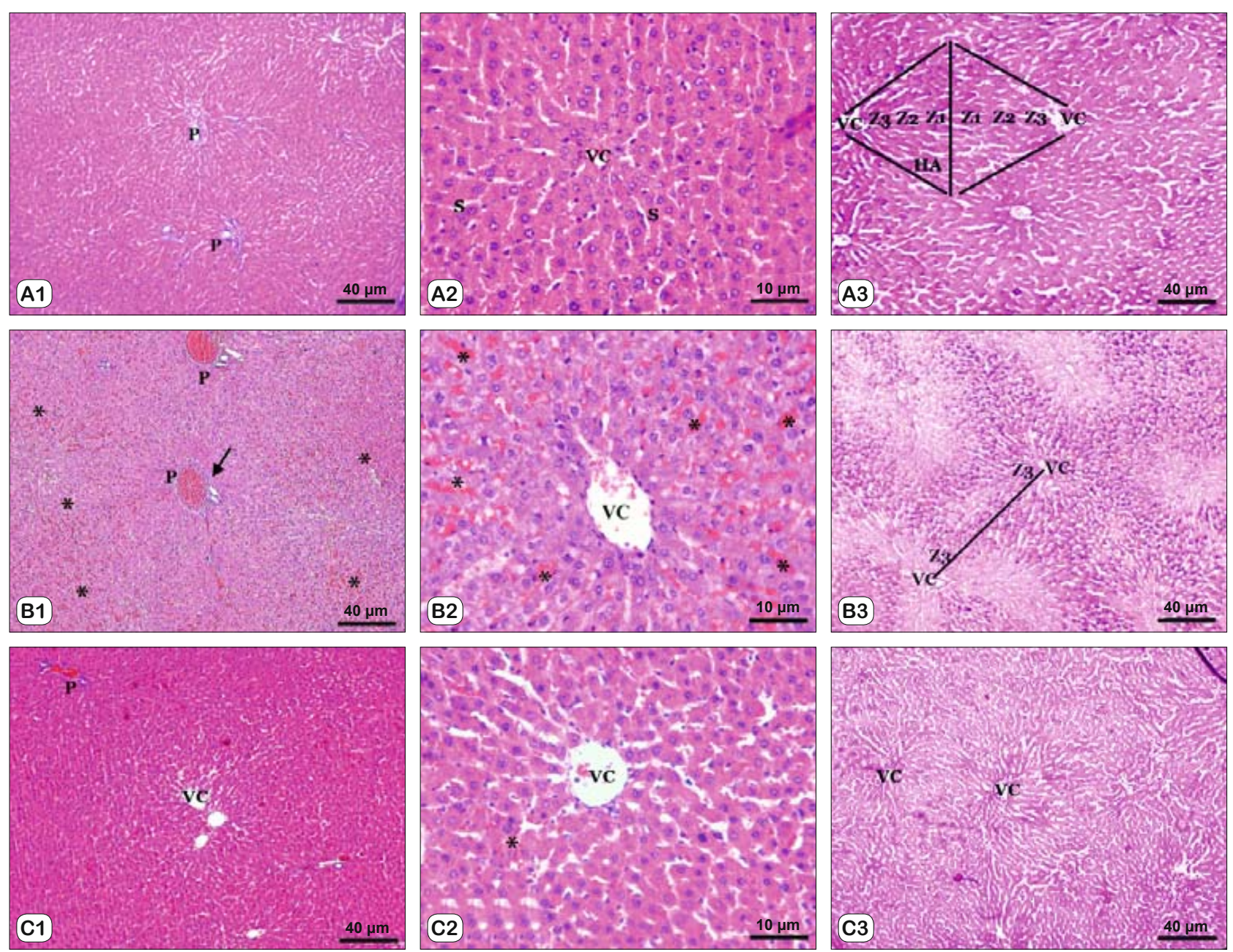

Fig. 1. This panel of the rat liver is stained by hematoxylin and eosin (1st and 2nd micrographs of each group on the left two columns of the panel) and Periodic Acid-Schiff reaction (3rd micrograps of each group on the right column of the panel). Micrograph on the first line is Group I (control), B micrograph on the second line is Group II (ischaemia-reperfusion), C micrograph on the third line is Group III (etanercept infused ischemia reperfusion group). Fig. 1A micrograph shows the structure of liver composed of hepatocytes, portal tracts (P) which contain the main blood vessels, hepatic (centrilobular) venule " vena centralis" (VC) and the sinusoids (S). The hepatic acinus (HA) lies between two terminal hepatic venules and is divided into zones 1,2 and $3(\mathrm{Z1}, \mathrm{Z2}, \mathrm{Z3})$. Fig. 1B micrographs, inflammatory cell infiltration (arrow) in the portal tract and congestion in the parenchyma (*). Fig. 1C micrograph shows the treated groups in close morphology to the regular structure of liver except the mild congestion $\left(^{*}\right)$ in certain regions.

cytoplasm were markedly reduced especially near the central vein (Fig. 3B). There was congestion in many areas because of the breakdown of continuous sinusoidal lining. The cytoplasm of the hepatocytes was vacuolated. Kupffer cells and the endothelial cells lining the sinusoids were disordered and the space of Disse was expanded (Fig. 3CD).

In Group III, the unit plasma membranes of the hepatocytes were observed and necrosis of the cells was markedly reduced. Hepatocytes were normal with their euchromatic nucleus, lysosomes and rich in rough endoplasmic reticulum close to the nuclear membrane and cytoplasm (Fig. 4A). The whole tissue was not dense and stranded as observed in the Group II. Sinusoidal lining cells were organised and erythroctes were seen within the lumen (Fig. 4B). The plasma membranes of adjacent hepatocytes close to canalicules were tightly bound and the intercellular space width was usual. Kupffer cells phagocyted the debris of inflammation and formed phagosomes (Fig. 4C). Portal tract containing terminal branch of the hepatic portal vein, terminal branches of the hepatic artery with the structure of arterioles and bile ductules presented no morphological alterations. Epithelial cells forming the ductuli biliferi were normal. Collagen formation at the periphery of the tract was observed (Fig. 4D).

\section{Discussion}

Basic research using animal models of ischemia reperfusion has elucidated dominant molecular pathways important in the pathogenesis of liver injury and inflammatory response is thought 

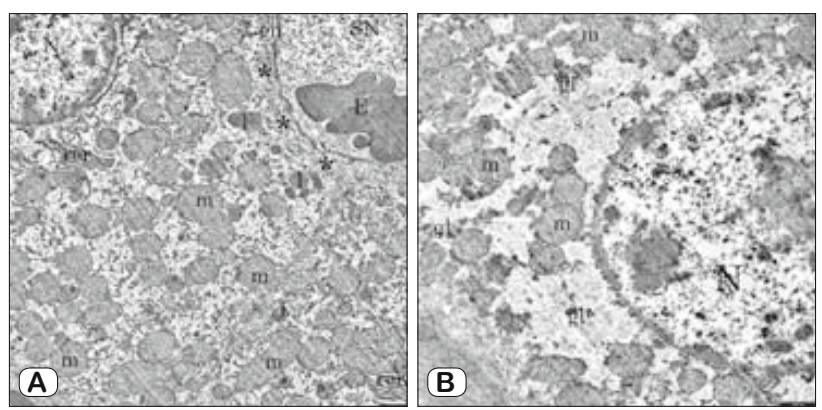

Fig. 2. Transmission electron micrographs of the control group. Fig. 2A, the regular appearance of liver section with adjacent hepatocytes through their nucleus $(\mathrm{N})$, lysosomes (l), mitochondria (m), and rough endoplasmic reticulums (rer). Transverse section of a sinusoid (SN) with an erythrocyte $(E)$ in its lumen is seen adjacent to the hepatocyte. The Disse space $(*)$ width is regular between the hepatocyte cell membrane and the endothel lining (en) sinusoid. Fig. 2B, the usual image of a hepatocyte cytoplasm with glycogen droplets and mitochondria.
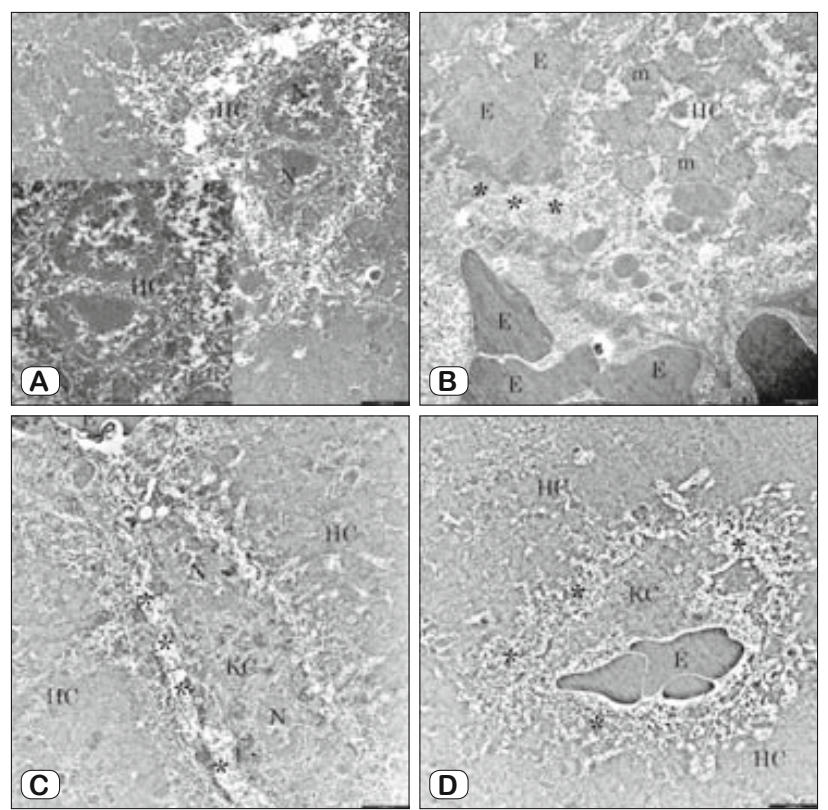

Fig. 3. Transmission electron micrographs of the ischemia reperfusion group. Fig. 3A, a binucleated $(\mathrm{N})$ hepatocyte $(\mathrm{HC})$ through necrosis and its higher magnification is seen. Fig. 3B,C,D, hepatocyte (HC) with mitochondria $(\mathrm{m})$ adjacent to sinusoids filled with erythrocytes (E), binucleated (N) Kupffer Cell (KC) with lysosomes (I) and dilated Disse Space $(*)$ are shown.

to have accountable response associated with this injury. The liver functions as an organ of the immune system through the function of the Kupffer cells that line the sinusoids, capable of migrating, phagocytosing large particles and also releasing inflammatory mediators (10). TNF- $\alpha$ is one of the cytokines released by Kupffer cells and hasan initiator role in the inflammation cascade, therefore hepatic injury. The potential of antagonizing TNF- $\alpha$ as a therapeutic strategy for hepatic IR was supported by our experimental ultrastructural study, in which we showed that etanercept protected the liver from IR injury by supressing inflammation.
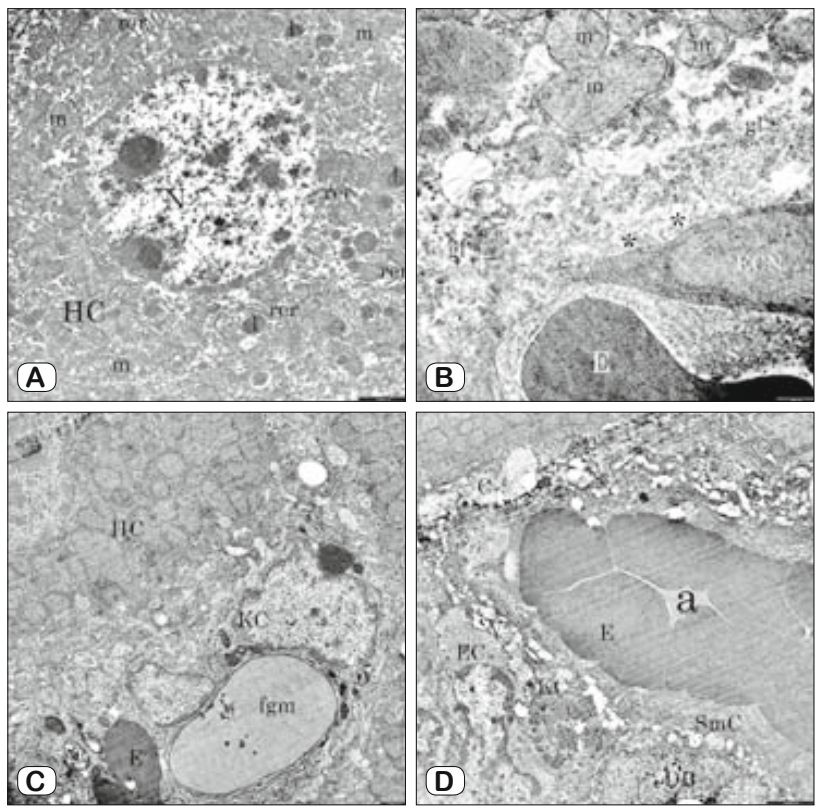

Fig. 4. Transmission electron micrographs of the etanercept injected ischemia reperfusion group. Fig. 4A, the regular structure of hepatocyte with nucleus $(\mathrm{N})$, mitochondrion $(\mathrm{m})$, rough endoplasmic reticulum (rer), lysosomes (l) and glycogen granules (gl) are seen. Fig. 4B, endothelial cell lining the sinusoid and its elongated nucleus (ECN) together with Disse Space $\left(^{*}\right)$ are shown. Fig. 4C, adjacent hepatocytes (HC), erytrocyte (E), Kupffer cell (KC) with phagocyted large material (fgm) are seen. Fig. 4D, arteriae interlobulares (a) with erythrocytes (E) in lumen and surrounding smooth muscle cell (SmC) are shown. Epithelial cells (EC) of ductuli biliferi interlobulares (DB) are seen. Kupffer cell (KC) cytoplasm is seen.

Anti-inflammatory cytokines have beneficial effects due to reduced activation of inflammatory cells and reduced microvascular damage. IL-6 is thought to act through several different mechanisms. IL- 6 can downregulate TNF- $\alpha$ mRNA during reperfusion, and it promotes hepatocyte regeneration (11). IL-10 has protective effect that is related to its ability to suppress the transcriptional activation of TNF formation, which may be responsible for the subsequent effects, such as reduced chemokine formation, reduced accumulation of neutrophils, and less ICAM-1 expression (12). IL-13 has a similar anti-inflammatory mechanism (13). Thus a complex network of regulatory cytokines and other mediators modulates the inflammatory response after hepatic ischemia.

In the present study, we evaluated the biochemical analyses by measuring the plasma AST and ALT levels. Anti TNF- $\alpha$ treatment significantly ameliorated these parameters in both treated groups.

Oxidative stress most likely plays a major role in the early development of liver injury and several experimental animal models show a beneficial effect of anti-oxidative drugs. Therapy with antioxidants (such as nitric oxide, tetrandine, L-arginin, allopurinol) administered intravenously has been investigated in a prospective double-blind placebo controlled randomized trial in patients with predicted severe AP but no effect on mortality could be demonstrated. In the present study, we evaluated the oxidative stress by measuring the plasma levels of MDA, GSH-Px and liver tissue 
601-607

levels of MDA and GSH-Px. Anti TNF- $\alpha$ agent also significantly decreased the oxidative stress in both treated groups.

Induction of heat shock proteins (HSP), especially HSP70 and HSP32, has also been implicated in the mechanism of preconditioning. HSP induction can reduce the nuclear binding of proinflammatory transcription factors (14-16) and increase the antioxidant capacity of cells (17). Both effects may contribute to the reduced formation of TNF- $\alpha$ and an attenuated inflammatory response in preconditioned livers $(18,19)$.

Over the recent years, a surgical strategy known as ischemic preconditioning, has been developed to reduce hepatic IR injury. This strategy is thought to 1) attenuate the microvascular disorders and the inflammatory response associated with this process 2) reduce the increase in hepatic TNF mainly released from Kupffer cells, thus attenuating the liver injury following hepatic $\mathrm{I} / \mathrm{R}$, and 3) prevent the systemic release of liver-associated TNF. The large amount of beneficial effects was loaded to the inhibition of TNF (20).

Since the anti TNF agents, such as adalimumab, etanercept, infliximab, certolizumab, and golimumab, have been approved by the U.S. Food and Drug administration (FDA) they are used to treat several immunological conditions. Campanati et al found that etanercept could be more efficacious to reduce the risk of developing hepatic fibrosis than PUVA therapy, and this preventive effect could be related to its anti-inflammatory and glucose homeostatic properties. Correlatively, collagen synthesis in the $\mathrm{I} / \mathrm{R}$ group was further decreased in the etanercept treated rats in our study (21)

Yinghua Tian et al aimed to test the hypothesis that TNF $\alpha$ signaling plays a central role in the devolepment of liver injury and defective regeneration after the implantation of small liver grafts. They concluded that interruption of TNF $\alpha$ signaling or depletion of Kupffer cells improves survival after $30 \%$ of liver transplantations, reduces liver injury, and enhances regeneration (22).

However, it must be considered that TNF $\alpha$ is also involved in liver regeneration, which is vital for the long-term recovery from the ischemic insult and survival The complement cascade can be rapidly activated by the extensive release of cellular proteins during the early reperfusion period $(1,10)$. Hanson et al. indicated that TNF $\alpha$ is an integral proinflammatory cytokine that is responsible for modulating intracellular pathogens and granuloma formation. Because of TNF $\alpha$ is critical in immune defense against infections and contributes to the development of certain cancers, safety monitoring and management should be an important part of of these agents use (7).

\section{Conclusion}

Hepatic ischemia reperfusion injury is a pathology that can lead to cell injury that is aggravated by the activation of Kupffer cells, formation of proinflammatory mediators and reactive oxygen species from various intracellular sources. Therefore, it isn't easy to achieve effective protection by targeting individual mediators or mechanisms. In contrast, a promising protective strategy against ischemia-reperfusion injury explored during the last few years is suppressing the formation of TNF- $\alpha$ or neutralizing it with antibodies.

Future evaluations of the potential role of etanercept in the treatment of hepatic surgery patients should also take into consideration its cost-effectiveness, which may vary across countries, and associated level of patient satisfaction. In conclusion, etanercept significantly reduced ischemia reperfusion hepatic injury in vivo. This study not only explored the molecular mechanism underlying the therapeutic effect of etanercept on IR, but also provided an ultrastructural microscopic evidence for the interaction between TNF- $\alpha$ and etanercept.

\section{References}

1. Jaeschke H. Molecular mechanisms of hepatic ischemia-reperfusion injury and preconditioning. Am J Physiol Gastrointest Liver Physiol 2003; 284 (1): 15-26.

2. Jaeschke H, Bautista AP, Spolarics Z, Spitzer JJ. Superoxide generation by neutrophils and Kupffer cells during in vivo reperfusion after hepatic ischemia in rats. J Leukoc Biol 1992; 52: 377-382.

3. Jaeschke H, Farhood A, Bautista AP, Spolarics Z, Spitzer JJ. Complement activates Kupffer cells and neutrophils during reperfusion after hepatic ischemia. Am J Physiol Gastrointest Liver Physiol 1993; 264 : 801-809.

4. Vandenabeele P, Declercq W, Beyaert R, Fiers W. Two tumour necrosis factor receptors: structure and function. Trends Cell Biol 1995; 5: 392-399.

5. Beyaert R, Cuenda A, Vanden Berghe W, Plaisance S, Lee JC, Haegeman G, Cohen P, Fiers W. The p38/RK mitogen-activated protein kinase pathway regulates interleukin-6 synthesis response to tumor necrosis factor. EMBO J 1996; 15 (8): 1914-1923.

6. Shuh M, Bohorquez H, Loss G, Cohen AJ. Tumor Necrosis Factor-a: Life and Death of Hepatocytes. During Liver Ischemia/Reperfusion Injury. Ochsner J 2013; 13: 119-130.

7. Hanson RL, Gannon MJ, Khamo N, Sodhi M, Orr AM, Stubbings J. Improvement in safety monitoring of biologic response modifiers after the implementation of clinical care guidelines by a specialty. J Manag Care Pharm 2013; 19 (1): 49-67.

8. Chastre A, Bélanger M, Beauchesne E, Nguyen BN, Desjardins P, Butterworth RF. Inflammatory cascades driven by tumor necrosis factor-alpha play a major role in the progression of acute liver failure and its neurological complications. PLoS One 2012; (11): e49670. doi: 10.1371/ journal.pone.0049670.

9. Lowry OH, Rosebrough NJ, Farr AL, Randall RJ. Protein measurement with folin phenol reagent. J Biol Chem 1951; 182: 265-275.

10. Wisse E, Braet F, Luo D, De Zanger R, Jans D, Crabbé E, Vermoesen A. Structure and Function of Sinusoidal Lining Cells in the Liver. Toxicol Pathol 1996; 24 (1): 100-111.

11. Camargo CA Jr, Madden JF, Gao W, Selvan RS, Clavien PA. Interleukin-6 protects liver against warm ischemia/reperfusion injury and promotes hepatocyte proliferation in the rodent. Hepatology 1997; 26 : $1513-1520$.

12. Yoshidome H, Kato A, Edwards MJ, Lentsch AB. Interleukin-10 suppresses hepatic ischemia/reperfusion injury in mice: implications of a central role for nuclear factor kappaB. Hepatology 1999; 30: 203-208. 
13. Yoshidome H, Kato A, Miyazaki M, Edwards MJ, Lentsch AB. IL13 activates STAT6 and inhibits liver injury induced by ischemia/reperfusion. Am J Pathol 1999; 155: 1059-1064.

14. Serafín A, Fernández-Zabalegui L, Prats N, Wu ZY, Roselló-Catafau J, Peralta C. Ischemic preconditioning: tolerance to hepatic ischemiareperfusion injury. Histol Histopathol 2004; 19 (1): 281-289.

15. Kume M, Yamamoto Y, Saad S, Gomi T, Kimoto S, Shimabukuro T, Yagi T, Nakagami M, Takada Y, Morimoto T, Yamaoka Y. Ischemic preconditioning of the liver in rats: implications of heat shock protein induction to increase tolerance of ischemia-reperfusion injury. J Lab Clin Med 1996; 128: 251-258.

16. Redaelli CA, Tian YH, Schaffner T, Ledermann M, Baer HU, Dufour JF. Extended preservation of rat liver graft by induction of heme oxygenase-1. Hepatology 2002; 35: 1082-1092.

17. Uchinami H, Yamamoto $Y$, Kume M, Yonezawa K, Ishikawa $Y$, Taura K, Nakajima A, Hata K, Yamaoka Y. Effect of heat shock preconditioning on NF-kappaB/I-kappaB pathway during I/R injury of the rat liver. Am J Physiol Gastrointest Liver Physiol 2002; 282: 962-971.
18. Bauer M, Bauer I. Heme oxygenase-1: redox regulation and role in the hepatic response to oxidative stress. Antiox Redox Signal 2002; 4: 749-758.

19. Arai M, Thurman RG, Lemasters JJ. Ischemic preconditioning of rat livers against cold storage-reperfusion injury: role of nonparenchymal cells and the phenomenon of heterologous preconditioning. Liver Transpl 2001; 7: 292-299.

20. Yonezawa K, Yamamoto Y, Yamamoto H, Ishikawa Y, Uchinami H, Taura K, Nakajima A, Yamaoka Y. Suppression of tumor necrosis factor-alpha production and neutrophil infiltration during ischemia-reperfusion injury of the liver after heat shock preconditioning. J Hepatol 2001; 35: 619-627.

21. Campanati A, Ganzetti G, Sario AD, Damiani A, Sandroni L, Rosa L, Benedetti A, Offidani A. The effect of etanercept on hepatic fibrosis risk in patients with non-alcoholic fatty liver disease, metabolic syndrome, and psoriasis. J Gastroenterol 2012 (10).

22. Tian Y, Jochum W, Georgiev P, Moritz W, Graf R, Clavien PA. Kupffer cell-dependent TNF-alpha signaling mediates injury in the arterialized small-for-size liver transplantation in the mouse. Proc Natl Acad Sci USA 2006; 103 (12): 4598-4603. 